\title{
The co-evolution of two Chinese mobile short video apps: Parallel platformization of Douyin and TikTok
}

Kaye, David Bondy Valdovinos ; Chen, Xu ; Zeng, Jing

\begin{abstract}
TikTok is the international twin of China's mobile short video app, Douyin, and one of the fastest growing short video platforms in the world. Owned by Chinese tech giant, ByteDance, TikTok and Douyin share many similarities in terms of appearance, functionality, and platform affordances; however, they exist in radically different markets and are governed by radically different forces. Unlike other popular mobile media platforms in China and internationally, TikTok and Douyin are neither part of the big three tech giants in China nor the big five in the US. This provides an interesting case study to investigate how an emerging internet company adapts its products to better fit divergent expectations, cultures, and policy frameworks in China and abroad. Using the app walkthrough method informed by platformization of culture production theory, this study highlights the similarities and distinctions between these two platforms. We argue the co-evolution of Douyin and TikTok is a new paradigm of global platform expansion that differs from strategies of regionalization adopted by previous major social media platforms. We contribute to platformization theory by developing the concept of parallel platformization to explain ByteDance's strategies for surviving in two opposing platform ecosystems in China and abroad.
\end{abstract}

DOI: https://doi.org/10.1177/2050157920952120

Posted at the Zurich Open Repository and Archive, University of Zurich ZORA URL: https://doi.org/10.5167/uzh-190628

Journal Article

Accepted Version

Originally published at:

Kaye, David Bondy Valdovinos; Chen, Xu; Zeng, Jing (2021). The co-evolution of two Chinese mobile short video apps: Parallel platformization of Douyin and TikTok. Mobile Media Communication, 9(2):229-253.

DOI: https://doi.org/10.1177/2050157920952120 


\title{
The co-evolution of two Chinese mobile short video apps: Parallel platformization of Douyin and TikTok
}

\author{
D. Bondy Valdovinos Kaye \\ Queensland University of Technology, Australia \\ Xu Chen
}

Xiamen University, China

Jing Zeng

University of Zurich, Switzerland

\begin{abstract}
TikTok is the international twin of China's mobile short video app, Douyin, and one of the fastest growing short video platforms in the world. Owned by Chinese tech giant, ByteDance, TikTok and Douyin share many similarities in terms of appearance, functionality, and platform affordances; however, they exist in radically different markets and are governed by radically different forces. Unlike other popular mobile media platforms in China and internationally, TikTok and Douyin are neither part of the big three tech giants in China nor the big five in the US. This provides an interesting case study to investigate how an emerging internet company adapts its products to better fit divergent expectations, cultures, and policy frameworks in China and abroad. Using the app walkthrough method informed by platformization of culture production theory, this study highlights the similarities and distinctions between these two platforms. We argue the coevolution of Douyin and TikTok is a new paradigm of global platform expansion that differs from strategies of regionalization adopted by previous major social media platforms. We contribute to platformization theory by developing the concept of parallel platformization to explain ByteDance's strategies for surviving in two opposing platform ecosystems in China and abroad.
\end{abstract}

\section{Keywords}

Douyin, TikTok, platformization, mobile apps, short video

\section{Introduction}

Around the world, the year 2019 may be remembered as the year adults frantically sought to figure out what a "TikTok" was and why millions of teens were so obsessed with it.

\section{Corresponding author:}

Xu Chen, School of Journalism and Communication, Xiamen University, Siming South Road 422, Xiamen, Fujian 361005, China.

Email: hichenxu@hotmail.com 
In 2019, TikTok shot to global prominence seemingly overnight and cemented itself alongside touchstone digital media platforms like Facebook and WhatsApp both through its popularity in massive markets like the US and India, but also through its popularity in the largest internet market in the world: China (Chen, 2019; Savic \& Albury, 2019).

TikTok is the international version of China's mobile short video platform, Douyin, owned by the Chinese tech giant, ByteDance. Both platforms have seen sustained growth from 2019 into 2020, surpassing 2 billion downloads in April 2020 amid an ongoing global health crisis $(\mathrm{Qu}, 2020)$. Douyin and TikTok are both short video platforms that feature 15 to 60 second videos, largely constituted of user-generated content (UGC) as opposed to professionally generated content (PGC). This study extends recent inquiries into Chinese digital spaces for platformization theory building (de Kloet et al., 2019; Lin \& de Kloet, 2019; Wang \& Lobato, 2019; Zhang, 2020) by exploring and comparing the development of two short video platforms, Douyin and TikTok, in China and abroad.

"Short video" refers to video content that is shorter than 5 minutes distributed via digital media platforms. Short video features include low-cost production, highly spreadable content, and blurry boundaries between producers and consumers (Kong, 2018). By October 2018, short video platforms in China reached 648 million users, accounting for almost $80 \%$ of all internet users in the country (China Internet Network Information Centre [CNNIC], 2019). China has developed a "self-sustained social media ecosystem" (McLelland et al., 2017, p. 59) due to the restrictions of the "Great Firewall" on the internet of mainland China. In this rapidly growing and self-sustained social media ecosystem, the emergence of copycat (shanzhai) versions of Western social media in mainland China have sparked the development of China's own unique social media applications (Keane \& Zhao, 2012). Chinese shanzhai platforms have become improved and localized (McLelland et al., 2017), and have developed different trajectories in China. What has led social media development along distinctive paths in China is "local dynamics, individual agency, and indigenous innovation in domesticating Western technologies and concepts" (McLelland et al., 2017, p. 64). The birth and growth of the short video industry in China and later in the West is a powerful example of how platforms and platform economies may develop differently along two parallel paths.

China's burgeoning short video industry includes various platforms dedicated to social entertainment, news and information, and video editing (Kong, 2018). Kuaishou, Douyin, and Meipai are three major players among those short video platforms tailored for social entertainment (Su, 2019). The growth of international short video industries has progressed slower than the Chinese short video industry (Su, 2019). For example, one early international entrant, Vine, received great interest and was acquired by Twitter before its launch in 2012 (Isaac, 2016). While Twitter would go on to shut down Vine in 2016, the legacy of short video platforms persisted in apps such as Snapchat, Musical.ly, and later Facebook and Instagram "story" functions. TikTok's predecessor, Musical.ly was launched by a Chinese tech company that targeted the US market in 2014 (Spangler, 2016). After its success in the US, Douyin's parent company ByteDance acquired Musical.ly and rebranded it as TikTok in 2018 (Chen, 2019). TikTok shares similar digital architecture with Douyin but is marketed to a completely different user base and governed by different forces. TikTok's international user base was established in its earlier days as "Musical.ly", a primarily music lip-synching short video platform. Musical.ly reportedly had over 200 million subscribers in the US when it was acquired by ByteDance and rebranded it as TikTok (Dave, 2018). The decision to acquire Musical.ly was a strategic move by ByteDance that "combined [Douyin's] AI-fed streams and monetization track record with Muscal.ly's product innovation and grasp of users' needs and tastes in the West" (Fannin, 2019, p.1). 
TikTok and Douyin are exceptional cases to refine conceptual frameworks of platformization because they are owned by the same Chinese company but operated as two separate platforms in China and the rest of the world. TikTok and Douyin are unique considering the dominance of American and Chinese platform ecosystems across the globe (van Dijck et al., 2018). Douyin is not owned or aligned with the trio of Chinese internet giants, Baidu, Alibaba, and Tencent (BAT) and neither is TikTok associated with any of the "big five" internet companies: Google, Amazon, Facebook, Apple, and Microsoft (GAFAM). Previous studies have called for the decentralization of the US in scholarly platform debates (Steinberg \& Li, 2017) and to critically evaluate the technoorientalism that surrounds Chinese tech companies and platforms (de Kloet et al., 2019). Analyzing TikTok and Douyin side-by-side is instructive for future studies to examine platformization. TikTok and Douyin are developed by the same tech company but deployed in vastly different contexts and have thus far managed to survive as emerging platforms in two opposing but comparable oligopolistic platform ecosystems.

This article begins by looking at the ways the two apps are "platformized" in China and abroad (Nieborg \& Poell, 2018) followed by our method of data collection using the app walkthrough method (Light et al., 2018). We next organize our exploratory analysis and findings according to three dimensions of platformization: infrastructures, markets, and governance. We conclude by arguing that TikTok and Douyin evidence a strategy of parallel platformization that creates opportunities and challenges for ByteDance and other tech companies, Chinese or otherwise, looking to expand into new international markets. The main theoretical outcome of this study is our conceptualization of parallel platformization, which contributes to the growing scholarship theorizing platformization and has practical implications for understanding the intense and unfolding international political scrutiny facing TikTok and ByteDance.

\section{Conceptual framework: Platformization}

The term platformization was first introduced by Helmond (2015) to conceptualize the rise of digital platforms as the dominant infrastructural and economic model of the social web and its consequences. More recent scholarship further develops this concept to capture the growing complexity in wider technology ecosystems (Duffy et al., 2019; Nieborg \& Poell, 2018; van Dijck et al., 2018). Van Dijck and colleagues (2018) define platformization as the way in which entire societal sectors are transforming as a result of the mutual shaping that occurs between digital platforms and entities using platform infrastructures to provide products and services to end users. Focusing on digital video games as "contingent cultural commodities," Nieborg and Poell (2018, p. 4276) introduce platformization as a theoretical lens to investigate how economic, governmental, and infrastructural extensions of digital platforms are reshaping modes of production in the cultural industries. Theories of platformization are continuing to develop and call attention to the question of how cultural products and society are influenced by the penetration of platform logics or "the norms, strategies, mechanisms, and economies" of platforms (van Dijck \& Poell, 2013, p. 2). In this study, we define platformization as the process by which online applications shape cultural and social lives through their infrastructures, business models, and governance.

The concept platformization has been previously used to research the social implications of mainstream Western (Helmond, 2015; van Dijck et al., 2018), as well as Chinese (de Kloet et al., 2019; Lin \& de Kloet, 2019; Wang \& Lobato, 2019) digital platforms. We utilize platformization for a comparative analysis of ByteDance's two short video platforms. Chinese platforms have macro-level distinctions in terms of 
market, regulation, and affordances and attend to micro-level assumptions about platforms such as recommendation and personalization (Wang \& Lobato, 2019). These distinctive characteristics call for a "spatialized platform theory that is sensitive to the historical origins of particular platforms" to "de-Westernize" platform studies (Wang \& Lobato, 2019, p. 12). Platformization serves as an effective theoretical lens to analyze how the sharp contrasts between the technological and sociopolitical contexts in China and the Global "West" has partitioned TikTok and Douyin into two separate short video parallel universes.

Additionally, platformization provides a useful analytical lens through which we can systematically interrogate how platform logics and ecosystems affect the operation of cultural industries. Nieborg and Poell's (2018) framework highlights three analytical dimensions of platformization: infrastructures, markets, and governance. Beginning with infrastructures, platformization theory considers how features and services affect modes of digital cultural production and circulation. As Gillespie (2017) reasons:

Everything on a platform is designed and orchestrated. While social activity would exist without Facebook or Twitter the kind of social activities that occur there depend powerfully on the space and structure they provide. These structures are certainly not neutral: these are designed to invite and shape participation, toward particular ends (p. 258).

Gillespie (2017) underlines how platforms purposefully design their architecture to shape participation and discourse. Although Douyin and TikTok are both developed by the same company to provide essentially the same service, the distinctive cultural and socioeconomic ecosystem these two platforms operate may drive them to provide different platform infrastructure. We therefore ask:

RQ1: What are the main platform features distinguishing Douyin and TikTok, and their social implications?

Next, platformization considers the impact of dominant market structures in which platforms operate. Prior research on platformization underlines the impacts of multisided markets (Nieborg, 2015; Nieborg \& Poell, 2018) on economic exchanges taking place on platforms. Platforms serve, non-exclusively, as data-intermediaries that aggregate, facilitate, and control the connection between distinctive actors such as microentrepreneurs, institutions, content developers, advertisers, and end users (van Dijck et al., 2018, p. 38). The economic configuration of visual media platforms presents a similar form of multisided market.

Market structures of visual social media sites, such as Instagram, and video content platforms, such as YouTube, are interrelated with the "influencer economy" (Cunningham \& Craig, 2019). Visual media platforms depend on creative content uploaded by their users, particularly influencers, to attract traffic and generate revenue. User activities, social relations, and transactions are variously commodified, such as through targeted advertising. This form of commodification by platforms is described as "internet play labour [sic]" where users hardly feel like they are engaging in labor, but create economic value (Fuchs \& Trottier, 2013, p. 34). Influencers, as well as other micro-entrepreneurs, also rely on platforms to amplify their visibility, sell products, and receive monetary gifts from viewers directly (Zeng, 2020; Zhang et al., 2019). How platforms reward play labor varies from case to case and can significantly shape user practices. Because Douyin and TikTok target completely different contexts, the selfsustained Chinese market and international markets, the business models deployed to engage with play labor and generate revenues for creators may vary significantly. Thus, we ask:

RQ2. To what extent do Douyin and TikTok have different business models, and how do they impact platforms' and users' practices? 
Platform governance is the final dimension of platformization of cultural production theory. Platform governance seeks to identify the political economy of how cultural production is constructed, negotiated, and regulated through platforms (Nieborg \& Poell, 2018). Governance unpacks both the ways in which platforms are internally governed through terms of use (ToU), content moderation, and development guidelines as well as the larger governing or regulatory systems that externally influence platforms. Gillespie (2017) notes that governance of platforms and governance by platforms are two distinctive phases of governance that should be investigated separately. The former focuses on policies specifying the liabilities of online platforms; the latter refers to interventions conducted by platforms themselves to police content they host, for both legal and economic reasons (Gillespie, 2017).

Facing distinct regulatory environments at home in China and abroad, ByteDance's commercial success relies on responsive policies regulating TikTok and Douyin according to disparate systems of governance. Previous scholars argue that platform regulations in the West are underpinned by liberal democratic ideologies while those in China are underpinned by paternalistic ideologies with command-and-control regulations from the Chinese government (Wang \& Lobato, 2019). But more complex and co-regulatory governance frameworks have also been shown in China's dealings with digital platforms operated by Chinese tech giants such as Tencent and Alibaba (Hong \& Xu, 2019). Given the malleable nature of social media platforms (Bucher \& Helmond, 2017), more nuanced complexities are expected to emerge when it comes to regulations of and by TikTok and Douyin respectively. Therefore, we ask:

RQ3. How do Douyin and TikTok differ in terms of platform governance?

Collectively our three research questions seek to explore the differences and highlight the similarities in the platformization of TikTok and Douyin. To answer these questions, we systematically analyze Douyin and TikTok using the app walkthrough method and trade press analysis.

\section{Method}

We collected data through three sessions of side-by-side app walkthroughs of Douyin and TikTok in August and September 2019. An app walkthrough is "a way of engaging directly with an app's interface to examine its technological mechanisms and embedded cultural references to understand how it guides users and shapes their experiences," (Light et al., 2018, p. 882). The app walkthrough method provides an excellent baseline for further analysis of understudied Chinese platforms such as TikTok and Douyin (Wang \& Lobato, 2019). App walkthroughs can also pinpoint unexpected user practices or, in this case, the nuanced similarities and differences between two ostensibly identical apps. Two researchers collected walkthrough data by interacting with the homepage, discovery page, settings page, terms and conditions, and engaging with various features of both platforms such as video creation features, social features, cross-platform connectivity features and reporting features. Data were also collected from analyzing inapp settings, privacy policies, and ToU.

We structured our walkthrough according to platformization of cultural production theory (Nieborg \& Poell, 2018). First, to understand infrastructural differences and similarities, we explored the user interfaces and functions of both apps. Second, to explore business model distinctions we focused on in-app currency systems supplemented by trade press analysis. Third, to study governance, we examined trade press reports on governance of the two platforms and focused on terms of use, privacy settings, and reporting functions to understand governance by the two platforms. This 
conception of governance looks at the platforms themselves but also, in a broader sense, how ByteDance manages and regulates user activity according to specific operating models (Light et al., 2018). The three dimensions of platformization are theorized to be enmeshed and interconnected (Nieborg \& Poell, 2018). As such, while we organize our finding section according to infrastructures, business models (markets) and governance, we acknowledge areas overlap throughout the following sections.

\section{Findings}

\section{Infrastructures}

We first describe the differences and similarities of TikTok and Douyin's infrastructures, or platform features. At face value, the two platforms' features are very similar. These similarities cannot be solely explained by the fact that both platforms belong to the same genre of short video platforms. We noted design language (e.g., logo, aesthetics) and features (e.g., filming and uploading videos) shared by TikTok and Douyin that clearly reinforce the two platforms belong to the same parent company. Our walkthroughs revealed that the affordances of both platforms, in terms of user functionality, architecture and design (Nagy \& Neff, 2015) reflect the same logic. The design of the homepage is very similar, the two platforms have the same graphical user interface (GUI), and use the same logo displayed in the top left corner of videos, a vibrating red and blue musical note. The interface for creating short videos appears by tapping the "+" button that is located in the bottom middle of both apps (Figure 1 and Figure 2). The default setting of this video creation interface activates the device's front or rear camera with several setting options on the margins, such as "effects", "timer" and "filters". While the structure of this interface and names of options are identical on Douyin and TikTok, we found the default camera filter of Douyin makes users' skin tones appear much lighter than on TikTok. This speaks to a cultural distinction between the intended user base. In China, meibai (literally meaning "beautify whitening") is a widely popular camera tool (Li, 2019). Further cultural distinctions became more evident in exploring the "effects" settings where both apps display visual effects, filters, and stickers. The "effects" are predictably different on both apps as they reference aesthetics, popular culture, or internet memes that are only relevant to the respective local audiences. Given that cultural proximity is a key factor in mobile video consumption (Lin et al., 2015), different cultural references on Douyin and TikTok help the two platforms attract audiences.

We moved on to explore user experiences with infrastructures by assuming "a user's position while applying an analytical eye" (Light et al., 2018, p. 11). Our walkthrough of Douyin and TikTok from a user position revealed that core user infrastructures are nearly identical. To begin, the short video creation process on Douyin and TikTok is highly streamlined as both platforms include detailed and standardized templates to help users seamlessly become creators by capitalizing on popular trends. Both apps prioritize video creation, as illustrated by the central location of the video creation button on the main menu of the platforms (Figure 1 and Figure 2). There are two categories of maximum short video length on both platforms: 15 seconds and 60 seconds. Users on both platforms can upload video clips from their device or shoot video footage using their device camera. After uploading or shooting video clips, both platforms provide users with tools to edit the video, including "sounds", "effects", "text", "stickers", "volume", "trim", and "filters". While Douyin and TikTok share the same standardized processes for 
creating and posting videos, there is clear cultural variance in the video effects libraries (Figure 3 and Figure 4).

The final step in video creation is to prepare the video to post. Users on both platforms can write a caption for their video, add hashtags, and @ mention other users. They can select from a small variety of privacy settings and then can post their videos. In addition, both platforms display several other social media platforms on which users can share their videos. As we discuss below, the integrated platforms options differ based on Chinese and international social media use. The content creation processes on Douyin and TikTok are shaped by platform logics. While the seemingly neutral options offer users freedom and resources to create videos on the platform, these options actually shape content circulated on the platform into a range of certain forms. This streamlined content creation process would ensure the consistency of content on Douyin and TikTok respectively, in terms of video length, tones, aesthetics, and popular sounds.

Moving on to content consumption, the "home button" located in the bottom left corner of the on screen menu takes users to the main portal for viewing videos on both Douyin and TikTok: a full screen with the title of "For You" that automatically begins playing videos. Users cycle through videos by swiping up or down, and videos are recommended to users based on ByteDance's proprietary recommendation algorithm. Both viewing portals employ endless scrolling features for viewing and discovery. Users can scroll through videos for hours without ever getting to "the end" of the content and users can refresh the content feed at any time to see new algorithmically curated selections. Through this mechanism, every individual user will be shown unique videos in the "For You" section based on personalized user data. 


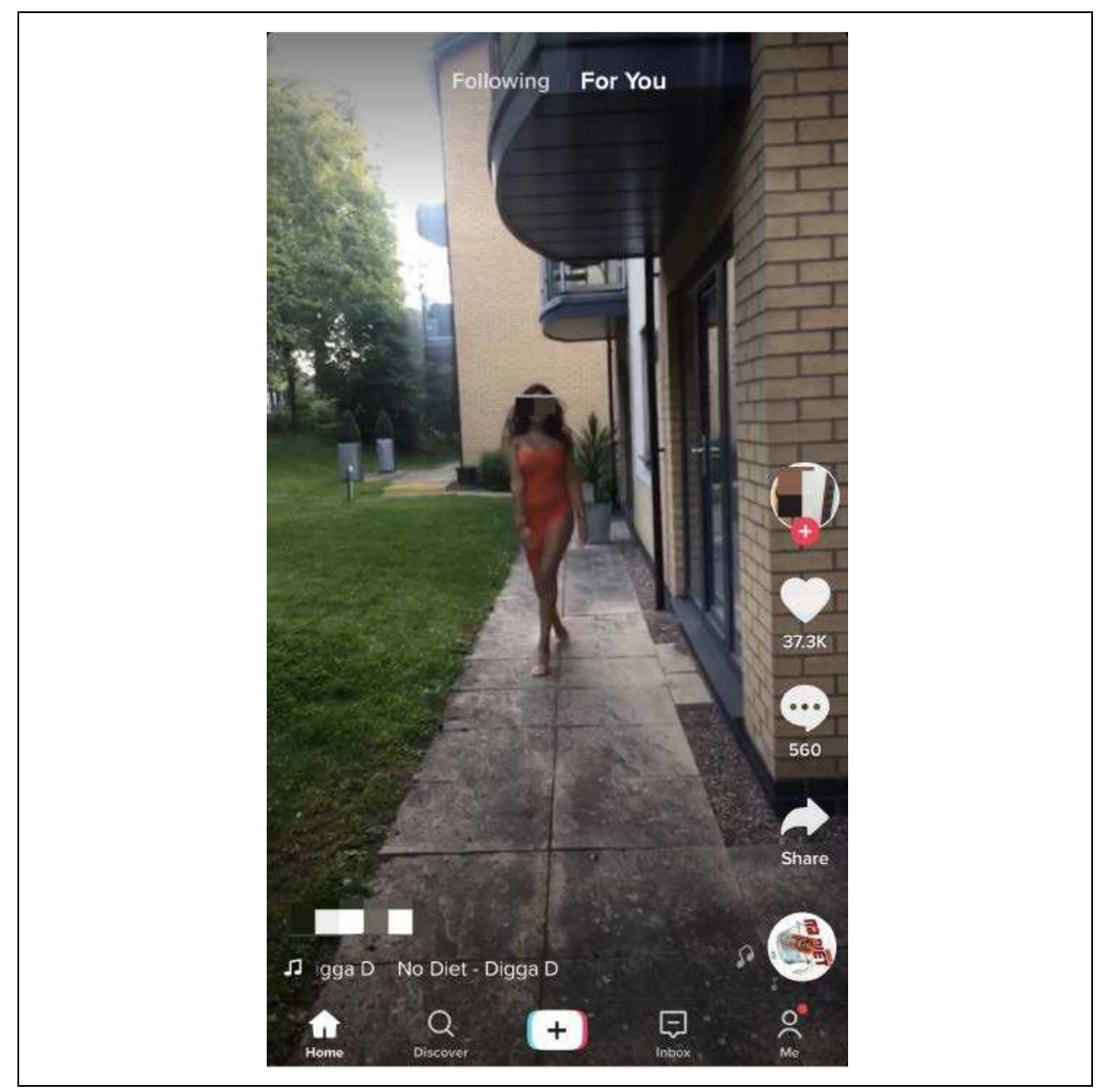

Figure 1. TikTok Home Screen.

The second main way to consume video content is the "Discover" interface, which shows a list of hashtags that are popular or trending. The Discover feature appears on both TikTok and Douyin and functions the same. Douyin also includes a second trending tab next to "Discover" entitled "positive energy" (Figure 5). "Positive energy (zheng nengliang)" is an ideological buzzword in China that is emblematic of Chinese patriotism (Du, 2014). Positive energy is an umbrella term that represents the ideologies, value systems, and ethos of the Chinese Communist Party (Yang \& Tang, 2018). Trending content on the "positive energy" trending page has been found to promote a specific type of propaganda, termed playful patriotism, that takes advantage of Douyin's platform features and audiences. Playful patriotism is "state-endorsed, but not necessarily orchestrated, light-hearted or amusing media content that promotes an established State ideology" (Chen et al., 2020, p. 15). There is no analogous feature to the "positive energy" trending tab on TikTok. 


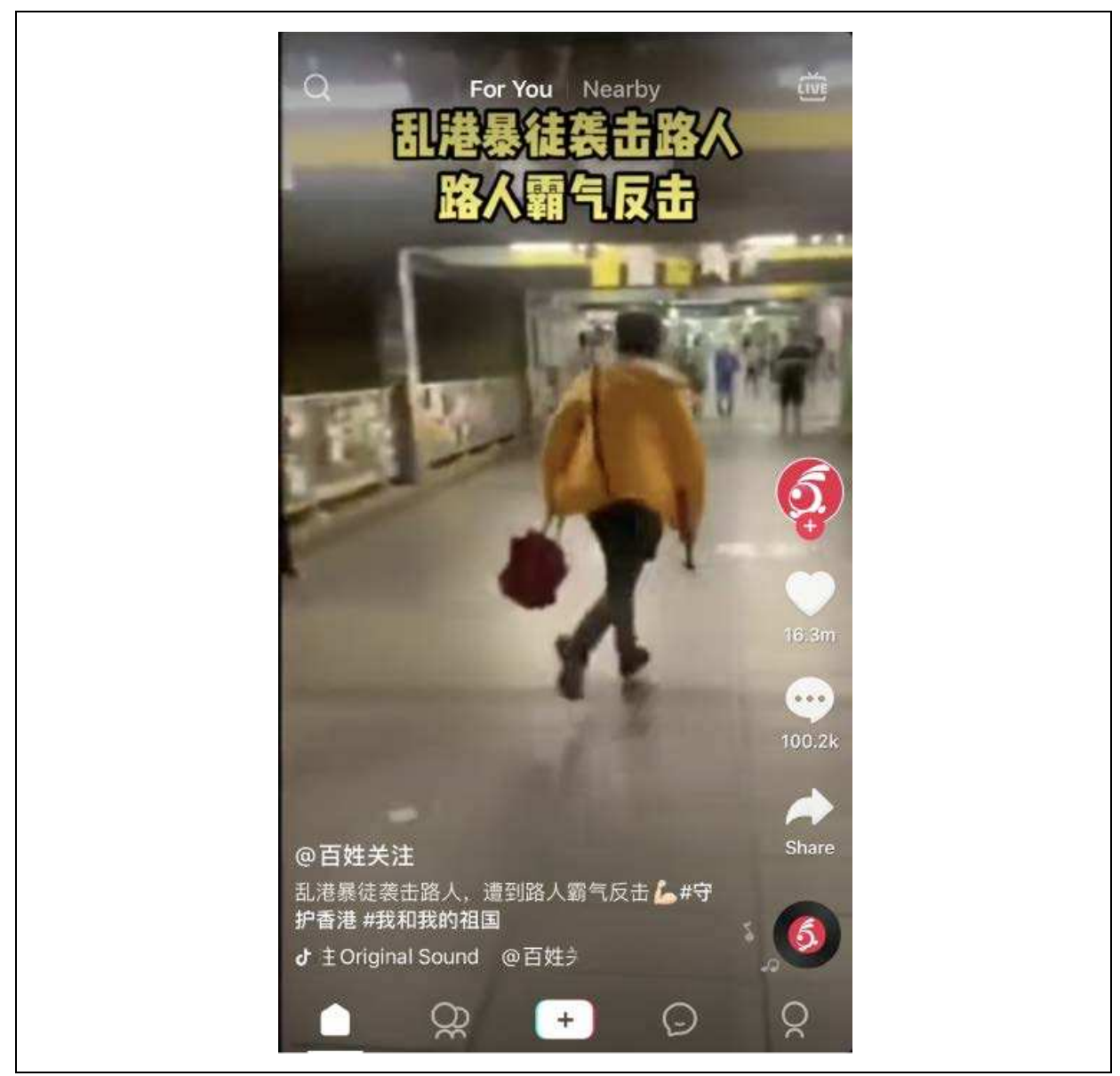

Figure 2. Douyin Home Page.

We noted a distinction in content consumption in terms of the web browser versions of Douyin and TikTok. Though our walkthrough primarily focused on the mobile version of both platforms, by attempting to access links to videos outside of the mobile platforms we found that Douyin has virtually no web browser support while TikTok takes a much more browser-friendly approach. There is currently no web browser to view or search for videos on Douyin. This strategic use of web browsers speaks to a distinction in China's mobile technological ecology (CNNIC, 2019). By disallowing web support, Douyin pushes users to its mobile app. TikTok, by contrast, has a browser platform that displays trending videos and users may log in to interact with content and embed TikTok videos on a variety of other international platforms. In April 2020, TikTok announced it would further expand its web browser features, indicating ongoing developmental support from ByteDance.

A final aspect of platform infrastructures we explored are social features. Both Douyin and TikTok include social features, such as sending friend requests, the ability to like or comment on videos, a messaging system, and cross-platform connectivity to 


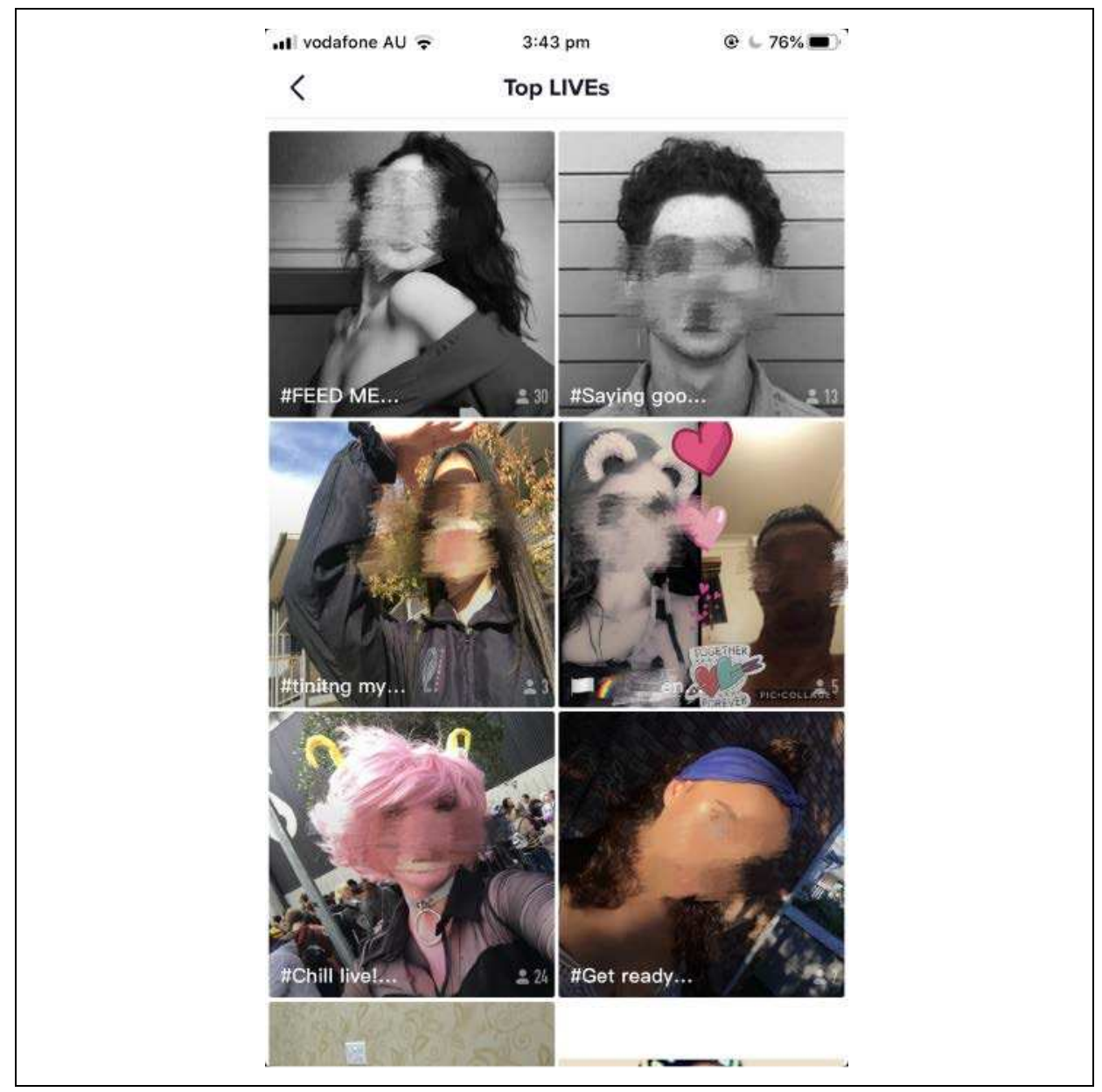

Figure 3. TikTok Top live streams.

share videos using other apps. Unsurprisingly, the cross-platform connectivity on Douyin is mainly designed to work with Chinese social media platforms, such as WeChat and Weibo, while TikTok is connected to more international platforms, such as Facebook, YouTube, Instagram, and Twitter. Videos shared on both platforms can be downloaded and shared in exactly the same manner. Users can also socially connect with each other through live streaming, but not all users can engage in live streaming. At the time of writing, live streaming features are only available to users with over 1000 followers on TikTok and 50,000 on Douyin. On Douyin the requirement for hosting live streaming also includes real-name verification of users. The mechanism of granting a limited number of vetted users access to the live streaming feature is similar to other Chinese short video apps, such as Kuaishou (Tan et al., 2020). Constructing live streaming services as a reward pushes users to create unique content more often to capitalize on fleeting trends. Creators are also incentivized to gain enough followers to live stream as streaming is currently the only way to make money directly through the platform on both Douyin and TikTok. 


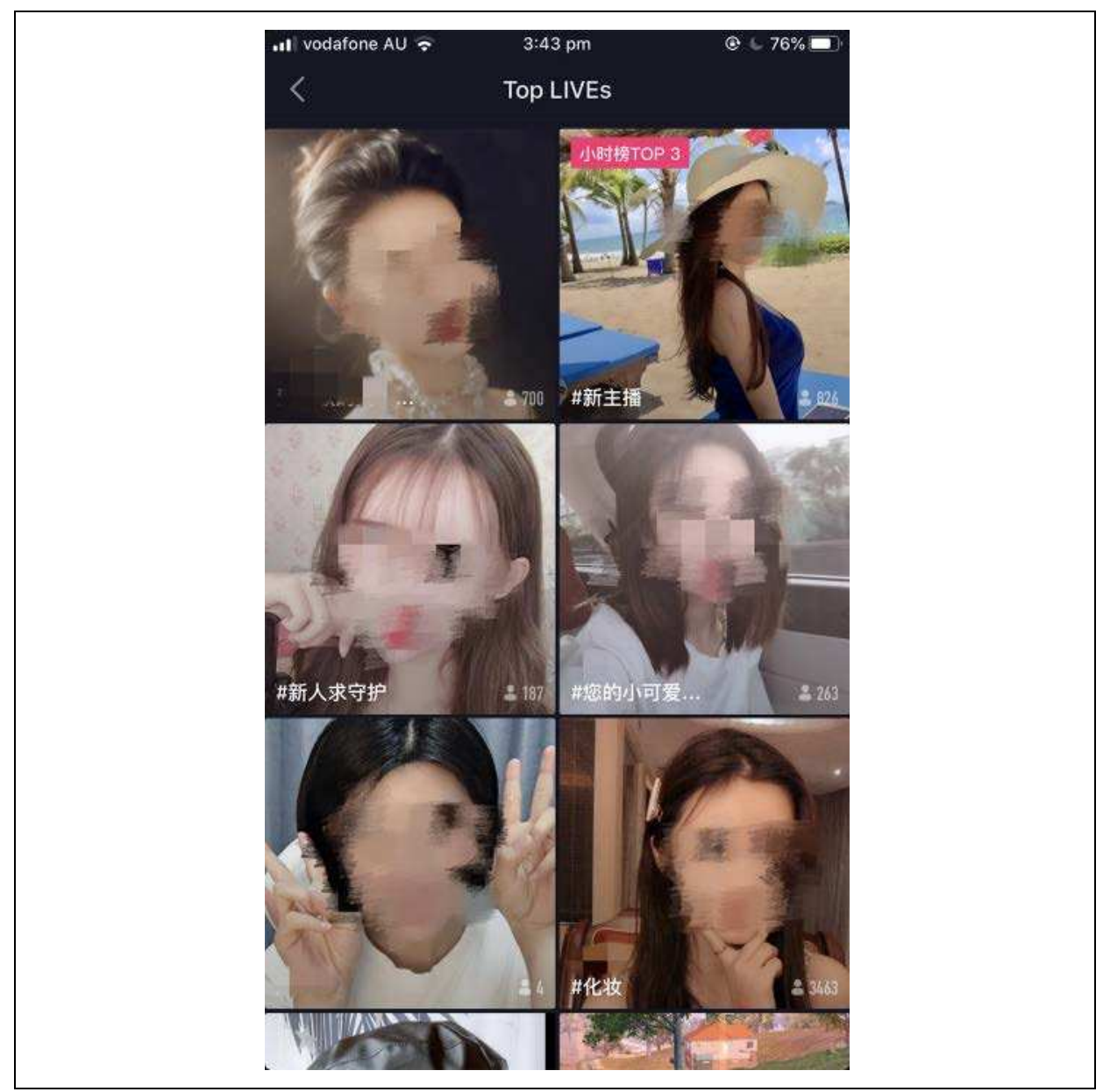

Figure 4. Douyin top live streams.

\section{Markets (business models)}

We next consider the similar business models that are evident on TikTok and Douyin, despite the two platforms existing in vastly different markets. On the one hand, in terms of the platforms themselves, revenues for TikTok and Douyin are heavily reliant on venture capital and advertising (Zhang, 2020). On the other hand, the only way for creators to directly earn money through both platforms is via a virtual currency system (Figure 6 and Figure 7). Virtual currency exists on both TikTok and Douyin where users can purchase coins to send as gifts to other users. Virtual gifting is nascent but also ubiquitous on Chinese live streaming platforms (Zhang et al., 2019). At time of writing, the only ways to give virtual gifts on both platforms is during live streams. On TikTok only users over the age of 18 can use virtual coins, according to the platform's latest policy on virtual gifting (TikTok, 2020). 


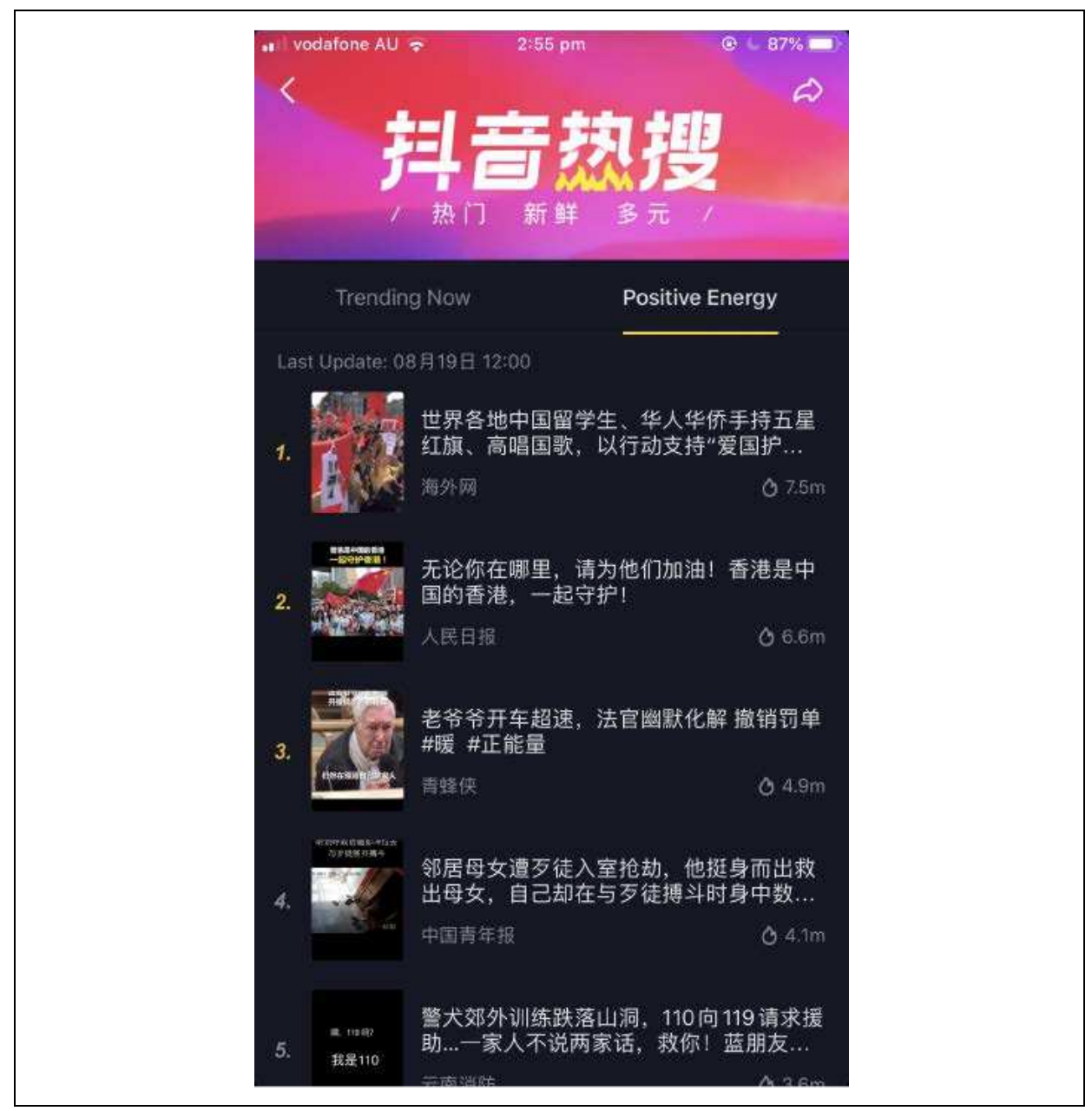

Figure 5. Douyin Positive Energy Page.

We found Douyin to have developed more mature business models than TikTok for creators. The short video market has had a longer gestation period in China (Su, 2019), which is reflected in Douyin's wider variety of options for direct monetization. In addition to virtual gifting, Douyin also includes a "merchandising on behalf" (daihuo) feature that embeds icons in live streams that link to products. Live stream audience members may click through the icon on the stream to purchase the product, earning revenue for the streamer. "Merchandising on behalf" allows live streamers to advertise multiple products sold by multiple brands during a single livestream. Merchandising on behalf was pioneered by Chinese online shopping platforms such as Taobao and Mogu (Yeung, 2019). This model has effectively turned viewers into buyers and now other international social media platforms, such as YouTube and Instagram, have integrated similar features (Yeung, 2019). 


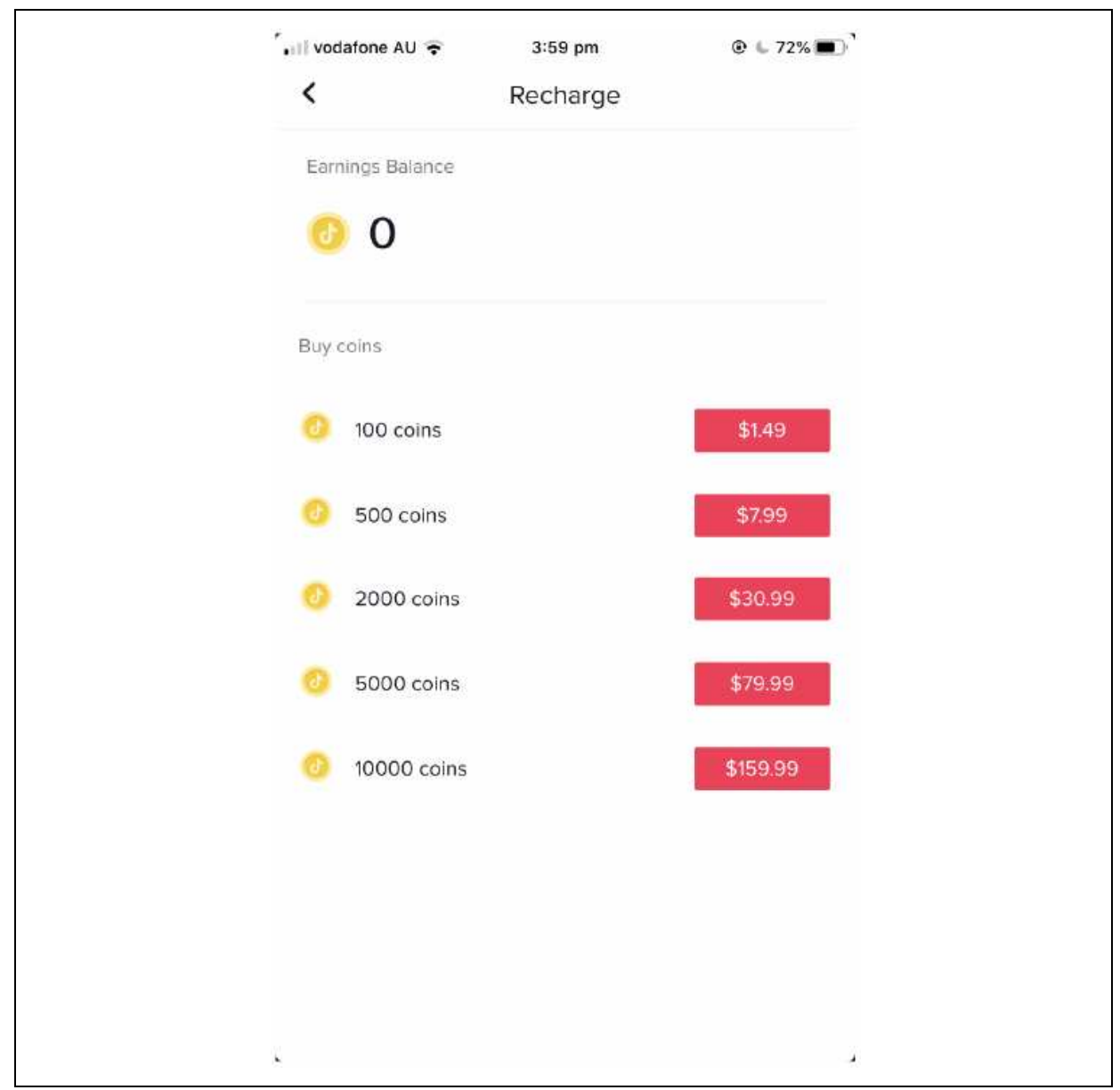

Figure 6. TikTok Virtual currency page.

TikTok creators, by contrast, rely much more on indirect revenue streams. Live streaming is not a pronounced feature of TikTok, with no dedicated section to view live streams exclusively. From our walkthrough, we noted users can find live streams by following creators who are streaming or by stumbling upon them while scrolling through "For You" or trending video pages, whereas Douyin features a dedicated live streaming section. This is significant as previous studies (Cunningham, Craig, \& Lv, 2019) highlight the cultural significance of live streaming in China as compared to international markets. Outside of live streaming, rising viral creators can leverage their success into influencer marketing agreements with brands (Meinsenzahl, 2019) while other creators use their TikTok profiles to funnel followers to other platforms, such as YouTube, with more options for monetization.

Overall, we find similar approaches to in-app monetization, which indicates the two platforms are still exploring and developing business models. Douyin has apparently benefited from the boom of China's social e-commerce market. China's virtual gifting 


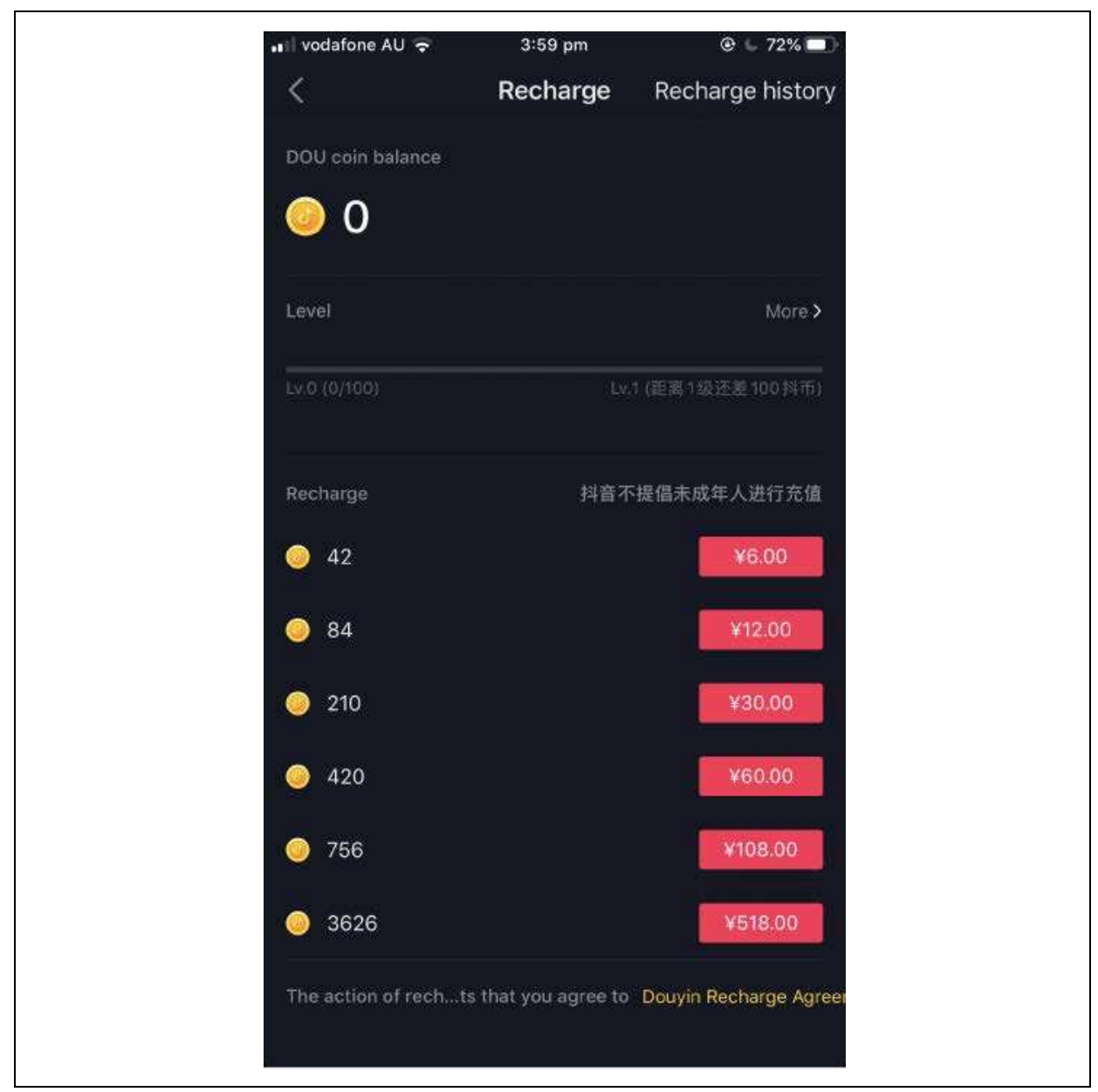

Figure 7. Douyin virtual currency page.

culture (Zhang et al., 2019) and live streaming shopping (Yeung, 2019) have allowed Douyin to apply existing successful business models to its platform. However, similar strategies are less effective on TikTok, due to the differences in international ecommerce markets. While content producers have become increasingly subject to the political economy of platforms (Nieborg \& Poell, 2018), emerging platforms are also subject to the political economy of the platform ecosystems in which they are embedded (Wang \& Lobato, 2019).

\section{Governance}

Finally, in analyzing platform governance, we consider the distinction between governance of platforms and governance by platforms (Gillespie, 2017). Governance of Douyin has been shaped by platform regulation forces in China while TikTok's platform governance has been shaped by numerous international regulatory forces. Digital platforms in China are required to comply with state imposed regulations (Wang \& Lobato, 2019). Although the Chinese government controls Chinese corporate platforms in indirect ways (van Dijck et al., 2018), the regime determines which political ideologies 
Chinese platforms should prioritize. Douyin's ToU indicates provisions that explicitly reference Chinese political ideologies. For example, one clause states users "must not overthrow socialist political system" (Douyin, 2019). Governance of TikTok has varied depending on national content. A major regulatory concern has focused on children and adolescents using the platform resulting in bans in countries like India (Parkin, 2019) and Indonesia (Silviana \& Potkin, 2018) and class-action lawsuits in countries like the US (Sherman, 2020). More recently governance action has involved concerns over privacy and national security, resulting in a second ban in India and threats of bans in the US and Australia (Taylor, 2020).

In responding to regulation and governance issues, ByteDance has employed similar platform responses in China and internationally. In 2018, a ByteDance platform called Neihanduanzi was ordered to shut down due to vulgarity. In response, ByteDance founder Zhang Yiming issued an apology ${ }^{1}$ and shortly thereafter a new trending section called "positive energy" was added to Douyin. The positive energy tab collects and displays a series of videos that promote a more playful style of patriotic content. This playful patriotism is "more akin to camouflage; ByteDance's survival tactic rather than the true aim of the platform," (Chen, Kaye, \& Zeng, 2020, p. 24). This tactic of responding to controversy by using the platform to promote social causes was repeated in India and Indonesia, two crucial markets for TikTok (Bellan, 2020; Chen, 2020). In Indonesia, TikTok was briefly banned for hosting "negative content" aimed at minors under the age of 18 (Silviana \& Potkin, 2018). A year later, TikTok was briefly banned in India for hosting pornographic material (Parkin, 2019). Both bans lasted less than two weeks but caused significant reputational damage to TikTok and ByteDance. In response, ByteDance announced coordinated education campaigns in Indonesia such as \#belajarbareng (study together) (Awaliyah, 2019) and a dedicated environmental campaign in India, \#cleanIndia (Sharma, 2019). Through these targeted campaigns, ByteDance responds to local authorities' governance of its platforms by encouraging TikTok and Douyin users to create content to repair the company's image.

Moving on to governance by TikTok and Douyin, a significant area of overlap involved content moderation. To moderate content, platforms rely on moderation algorithms, human moderators, users, or a combination of the three (Nieborg \& Poell, 2018). TikTok and Douyin both employ massive teams of human moderators as well as in-app reporting features for user-led moderation. Finding the reporting function was not intuitive on either platform. To access the reporting function, both TikTok and Douyin users must select the share icon and navigate to the report icon. The report option gives users a choice of several different violations they can indicate. At the time of our walkthrough, TikTok users could report content that depicts self-injury, harassment, bullying, pornography, violence or harm, spam, copyright infringement, hate speech, terrorism, promotion of drugs or weapons, and a category for "other". Douyin users had a wider array of reporting categories including pornography, political sensitivity, illegal/crime, spam/selling fake goods, rumor/swindling, insulting, not original content, copyright infringement, and other. Douyin's reporting function also featured a specific category for underage content that included inappropriate behavior, content that is not suitable for minors, discomfort, self-injury, and luring people to like, share, and follow.

Data-driven decision-making is critical in developing internal platform governance systems (van Dijck et al. 2018). By operating Douyin and TikTok in such divergent regulatory environments, ByteDance benefits from massive stores of user data from both platform ecosystems to help inform governance decisions. One example is that TikTok uses in-app governance to shape its content curation trending. Certain viral videos on Douyin will be selected to release on TikTok, and one selection criteria is that the Douyin 
video should be suitable for the local culture where TikTok operates. In doing so, TikTok can become more competitive in the international short video platform economy and ByteDance can, to some extent, bridge cultural flows between the two short video parallel universes.

\section{Discussion: Parallel platformization}

The features, functions, and everyday uses of TikTok and Douyin are nearly identical. The subtle distinctions between the two platforms, such as different visual effects to match audience preferences and privacy settings, do not change the fact they are, at facevalue, one and the same. Users can create, view, and share short videos in China with Douyin just as they can on TikTok internationally. But unlike the face-value similarities between other platforms, like iQiyi and Netflix (Wang \& Lobato, 2019), the waters of TikTok and Douyin flow from the same source into two highly distinct pools: the tightly controlled Chinese market and fiercely competitive international markets. In so doing, ByteDance has achieved a feat with these platforms that no previous major tech company, Chinese or American, has yet managed. Why was ByteDance able to crack the code?

One explanation is the format itself. Short video has been massively popular in China for several years, but slower to gain traction internationally. Facebook and Instagram arguably capitalized on short form video by launching "story" features, that enable users to create short videos or static slideshows that refresh daily. Snapchat enabled users to record short videos but was intended primarily as a video messenger with the added feature that videos delete themselves after watching. The principal analog to Douyin in the West besides Musical.ly was Vine, which was prematurely shuttered by Twitter in 2016. The recent boom in popularity of short video platforms internationally is evident by the foray of new competitors in recent years. Facebook launched its own short video platform Lasso in late 2018 and YouTube recently announced it was moving into the short video space with the eponymously named Shorts; even Vine made a comeback in 2020, rebranded as Byte.

We propose an alternate explanation for ByteDance's dual success with TikTok and Douyin, which lies in their platform practices, or the strategies, routines, experiences and expressions of creativity, that shape cultural production on platforms (Duffy et al., 2019, p. 2). Our analysis of TikTok and Douyin revealed unique and similar sets of platform practices, which we term parallel platformization.

Parallel platformization refers to practices of platforms that are developed by the same entity, offer nearly the same features, but differ in their infrastructures, governance, and market. We distinguish parallel platformization from platform localization or regionalization, in which developers adapt existing platforms to new markets (IndiaSA Comms Team, 2019; Perez, 2019). Parallel platformization extends Wang and Lobato's (2019) conception of a spatialized platform theory, by illustrating how these two platforms fundamentally function the same and share the same parent company but are governed vastly differently and exist in different markets.

The parallel platformization of TikTok and Douyin is significant because it is unprecedented. Several formal restrictions have prevented GAFAM platforms from gaining traction within China. The same formal restrictions do not necessarily apply to Chinese developers looking to expand outside of China, yet still powerful BAT platforms have struggled to find footing in Western international markets. As we note above, TikTok had a head start in the US market through its acquisition and rebranding of the already popular short video platform "Musical.ly". Even so, ByteDance's commercial 
success has relied on responsive practices, updates, and policies to adapt its platform to distinct markets. The following sections highlight some nuanced complexities that emerge in the parallel platformization of TikTok and Douyin.

\section{Circumscribed creativity}

At the infrastructure level, ByteDance has capitalized on the popularity and success of short video platforms in China to develop a platform that is carving out its own niche in international short video markets. A key aspect of success relates to how these platforms circumscribe creativity. Circumscribed creativity draws from the concept of circumscribed agency in media organizations. In media organizations that demand creative products, managers grant workers greater agency within the scope of pre-defined boundaries (Lotz, 2014). The effect of circumscribed agency can allow media workers to feel more freedom working in a normally more rigid organizational structure (Havens, 2014). In the same vein, circumscribed creativity refers to creative potential being shaped or guided by platformization. In this way, TikTok and Douyin empowers ordinary users to become creators by offering templates or guides that facilitate easy creation and capitalize on popular trends (Literat, 2018). Circumscribed creativity distinguishes TikTok and Douyin from other video creation and sharing platforms in the United States and China by including features that direct users to replicate popular formats for themselves.

TikTok and Douyin further circumscribe creativity by presenting users with suggestions for content creation via trending pages and hashtag search filters. While other scholars have observed digital creators display creative autonomy that is increasingly platform-agnostic (Mehta \& Kaye, 2019), the everyday uses and practices of TikTok and Douyin seem to foment the creation of certain types of mimetic content. Users can create videos based on the ones they were just watching by tapping to create a video that uses the same video filter, audio, or effects. This pushes passive users to become active creators with relative ease compared to other content creation platforms. In our walkthrough, we engaged in the video creation on both platforms and found that the infrastructures made the process extremely simple and straightforward, using just five taps of a finger. While content creation TikTok and Douyin is facilitated by circumscribed creativity, creators, particularly on TikTok (Hayes, 2020), remain vexed as to how they can monetize their videos.

\section{The gift economy}

Platform practices relating to markets, business models and monetization create friction between TikTok and Douyin. As we note, the only way to directly monetize in both platforms is through virtual currency and virtual gifting. This virtual economy, in turn, is only accessible via live streaming, which is currently gated by follower count on both platforms. This causes fewer issues in the Chinese market, as live streaming is wellestablished in China, as are conventions of virtual gifting (Cunningham, Craig, \& Lv, 2019). Douyin also makes it easier to find live streams with its dedicated live streaming section.

That does not mean that TikTok is having trouble developing business models or monetization strategies. There are several approaches detailed in the trade press to monetize TikTok popularity through influencer marketing, links to other monetized platforms, and merchandising. Sponsored content and brand partnerships are beginning to appear on TikTok but as one trade publication observes, "while the odd sponsored 
video appears occasionally, there isn't a clear-cut way for creators to monetize their content directly" (Wetmore \& Spritz, 2019, p. 1). ByteDance may change its approach to business models and monetization, though reports indicate the current strategy is working in China (Zhang, 2020). Further, ByteDance's recent valuation, estimated to be around $\$ 100$ billion (USD), indicates the company is not in dire financial straits (Sheng, 2020). This market-level tension highlights the distinction between the Chinese digital content market and international markets, but this tension will likely be more surmountable than governance issues ByteDance faces due to the stigma of being "a Chinese platform" abroad.

\section{Identity crisis}

Prior to acquiring and rebranding Musical.ly, ByteDance founder Zhang Yiming stated in an interview that he hoped ByteDance's core products would "be as border-less as Google" (Tung \& Zhang, 2017, p. 1); a lofty goal, even for one of the most successful Chinese tech companies not associated with BAT. However, despite attempts to distance itself from the Chinese government, ByteDance is a Chinese tech company and international audiences, regulators, and interest groups show little willingness to overlook that fact. Douyin clearly bears more marks of Chinese internet regulation than TikTok. As noted above, ByteDance has taken steps to appease the Chinese government, such as including a positive energy section following a domestic controversy (Chen, Kaye, \& Zeng, 2020). While officially TikTok does not include infrastructures or policies that can be directly related to the internet governance in mainland China, the platform has been the subject of intense scrutiny in different national contexts, in part due to its Chinese roots. This has thrown ByteDance's corporate identity into crisis amid challenges from different national governments.

In developing our conception of parallel platformization, we acknowledge the global standards in the nearly identical infrastructures of TikTok and Douyin, but call attention to the local standards by ByteDance in managing what is essentially the same platform operating in and subject to vastly divergent governance. For example, in December 2019, ByteDance reached a USD 1 million agreement to settle a class action lawsuit alleging TikTok was in violation of the US Children's Online Privacy Protection Act (Kelly, 2019). That same month, TikTok agreed to change its virtual currency policy in the UK responding to a probe by the BBC (Tidy, 2019). In March 2020, ByteDance announced it would no longer rely on moderation teams in China to moderate international content on TikTok (Fingas, 2020) and in May 2020 ByteDance appointed a new American CEO (Barnes \& Nicas, 2020). Even so, international media continue to report on several potential threats TikTok poses including privacy, cybersecurity (Kharpal, 2020), and ideology (Leskin, 2019). Previous studies have observed that "transnational platform companies tend to set global, rather than local standards," (Nieborg and Poell, 2018, p. 4285). Seemingly in acknowledgement, ByteDance founder Zhang Yiming announced in 2019 that the company would focus on creating globalized products with localized content (Digital Entertainment DreamWorks, 2019). Even so, the company remains besieged by international criticism and threats of bans at the time of writing (Taylor, 2020).

\section{Conclusion}

Douyin and TikTok have very similar digital infrastructures but they exist in different markets and are supported by different governance mechanisms. We argue ByteDance's 
domestic and international success with Douyin and TikTok is due to its unique and involved approach to platformization in China compared with international markets, which we term parallel platformization. In calling attention to the successes, such as employing nearly identical infrastructures that circumscribe creativity to increase engagement and popularity on both platforms, we also highlight the tensions associated with this process. At the market level, ByteDance appears to be clinging to a gift economy model of monetization, that relies predominately on live streaming and virtual currency exchange, that is more successful in Chinese markets than in international markets. At the governance level, ByteDance is struggling to adapt to heightened scrutiny in international contexts, as evidenced by its willingness to settle lawsuits and make changes to its platform governance policies to appease international critics and regulators. Through parallel platformization, ByteDance is positioned to strengthen its core products and further adapt them to divergent markets, while simultaneously being exposed to novel challenges and criticism. The big five Western tech giants, GAFAM, have yet to penetrate the Chinese market and the Chinese prophets of mass innovation, BAT, have simultaneously struggled to penetrate markets outside of China. As an outsider to both elite groups, ByteDance now has access to users in the largest short video markets in the world and millions of users both in China and in an array of international markets.

This study responds to a call for further research to "zoom in on contradictions, ambivalences, and connections" in Chinese digital spaces (De Kloet et al., 2019, p. 250). Further, we contribute to platformization theory-building by describing the parallel platformization of TikTok and Douyin in terms of platform practices (Duffy et al., 2019). Continued investigation is necessary to explore and contextualize changes implemented on TikTok and Douyin. Future research should explore the parallel platformization of other popular platforms, Chinese or otherwise, to strengthen or critique our findings here. Future studies should also consider parallel platformization of these two platforms in other markets, such as in India where TikTok is currently banned as a result of an escalating soft-power conflict between the Indian and Chinese governments as well as in markets such as the US and Australia that are threatening to take action against TikTok as part of an ongoing "political furore" (Taylor, 2020, p.1).

The parallel approach taken by ByteDance illustrates the outsider tech company's ability to transcend China's self-sustained social media ecosystem (McLelland et al., 2017) and thrive in more complex and unrestrained multi-sided markets (Nieborg \& Poell, 2018). We highlight areas of struggle, particularly in terms of markets and governance, as key areas for future research. Other Chinese tech companies, like short video competitor Kuaishou (Xue, 2020), are already vying to emulate ByteDance's successful parallel platformization of creating infrastructures to shape user experience and participation (Gillespie, 2017) while attempting to overcome challenges of being a Chinese app abroad. We expect there will be more examples of parallel platformization in the coming years and invite additional inquiry on whether such platforms will reinforce or disrupt notions of a binary platform ecosystem constituted by Chinese and American digital platforms.

\section{Funding}

The authors received no financial support for the research, authorship, and/or publication of this article.

\section{ORCID iD}




\section{Note}

1. See Zhang Yiming's apology letter (in Chinese) posted on Toutiao on April, 2018, https://www.toutiao.com/a1597389902009351

\section{References}

Awaliyah, G. (2019, December 6). Melalui \#BelajarBareng, TikTok Jadikan Belajar Lebih Seru. Republika. https://www.republika.co.id/berita/gaya-hidup/trend/19/12/06/q231o4328-melaluibelajarbareng-tiktok-jadikan-belajar-lebih-seru

Barnes, B. \& Nicas, J. (2020, May 18). Disney's head of streaming is new TikTok C.E.O. The New York Times. https://nyti.ms/2zaiHfV

Bellan, R. (2020, February 14). TikTok is the most downloaded app worldwide, and India is leading the charge. Forbes. https://www.forbes.com/sites/rebeccabellan/2020/02/14/tiktok-isthe-most-downloaded-app-worldwide-and-india-is-leading-the-charge/\#572695927266

Bucher, T., \& Helmond, A. (2017). The affordances of social media platforms. In J. Burgess, A. Marwick, \& T. Poell (Eds.), The SAGE handbook of social media (pp. 223-253). SAGE Publications.

Chen, W. (2020, January 15). Southeast Asians can now enjoy TikTok's videos on Netflix rival iflix. KrAsia. https://kr-asia.com/southeastern-asians-can-now-enjoy-tiktoks-videos-on-netflix-rivaliflix

Chen, X. (2019, April 1). TikTok is popular, but Chinese apps still have a lot to learn about global markets. The Conversation. https://theconversation.com/tiktok-is-popular-but-chinese-appsstill-have-a-lot-to-learn-about-global-markets-113039

Chen, X., Kaye, D. B. V., \& Zeng, J. (2020). \#PositiveEnergy Douyin: Constructing "playful patriotism" in a Chinese short-video application. Chinese Journal of Communication, 1-21. https://doi.org/10.1080/17544750.2020.1761848

China Internet Network Information Centre. (2019). The 44th Statistical Report on Internet Development in China. China Internet Network Information Centre. http://www.cnnic.net.cn/ hlwfzyj/hlwxzbg/hlwtjbg/201908/P020190830356787490958.pdf

Cunningham, S., \& Craig, D. R. (2019). Social media entertainment: The new intersection of Hollywood and Silicon Valley. University Press.

Cunningham, S., Craig, D., \& Lv, J. (2019). China's livestreaming industry: Platforms, politics, and precarity. International Journal of Cultural Studies, 22(6), 719-736. https://doi.org/10.1177/ 1367877919834942

Dave, P. (2018, August 2). China's ByteDance scrubs Musical.ly brand in favor of TikTok. Reuters. https://www.reuters.com/article/us-bytedance-musically/chinas-bytedance-scrubsmusical-ly-brand-in-favor-of-tiktok-idUSKBN1KNOBW

de Kloet, J., Poell, T., Guohua, Z., \& Yiu Fai, C. (2019). The platformization of Chinese society: Infrastructure, governance, and practice. Chinese Journal of Communication, 12(3), 249-256. https://doi.org/10.1080/17544750.2019.1644008

Digital Entertainment DreamWorks. (2019, November 6). The inflection point of the overseas version of TikTok? Нихiu. https://m.huxiu.com/article/324850.html

Du, S. (2014). Social media and the transformation of 'Chinese nationalism': 'Igniting positive energy' in China since the 2012 London Olympics. Anthropology Today, 30(1), 5-8. https://doi.org/10.1111/1467-8322.12083

Duffy, B. E., Poell, T., \& Nieborg, D. B. (2019). Platform practices in the cultural industries: Creativity, labor, and citizenship. Social Media + Society, 5(4), 1-8. https://doi.org/10.1177/ 2056305119879672

Fannin, R. (2019, September 13). The strategy behind TikTok's global rise. Harvard Business Review. https://hbr.org/2019/09/the-strategy-behind-tiktoks-global-rise 
Fingas, J. (2020, March 15). TikTok will stop using China-based moderators to screen foreign content. Engadget. https://www.engadget.com/2020-03-15-tiktok-to-stop-using-chinamoderators-for-foreign-content.html

Fuchs, C., \& Trottier, D. (2013). The Internet as surveilled workplayplace and factory. In S. Gutwirth, R. Leenes, P. de Hert, \& Y. Poullet (Eds.), European DATA Protection: Coming of age (pp. 33-57). Springer Netherlands.

Gillespie, T. (2017). Regulation of and by platforms. In J. Burgess, A. Marwick, \& T. Poell (Eds.), The SAGE handbook of social media (pp. 254-278). SAGE Publications.

Havens, T. (2014). Towards a structuration theory of media intermediaries. In D. Johnson, D. Kompare, \& A. Santo (Eds.), Making media work (pp. 39-62). NYU Press; JSTOR.

Hayes, R. (2020, April 5). How to make money on TikTok. Tech Junkie. https://www.techjunkie.com/make-money-tik-tok/

Helmond, A. (2015). The platformization of the web: Making web data platform ready. Social Media + Society, 1(2), 2056305115603080. https://doi.org/10.1177/2056305115603080

Hong, Y., \& Xu, J. (2019). Toward fragmented platform governance in China: Through the lens of Alibaba and the legal-judicial system. International Journal of Communication, 13(0), 4642-4662.

IndiaSA Comms Team. (2019, January 29). Uber puts safety at the heart of driver experience. Uber Newsroom. https://www.uber.com/en-IN/newsroom/uber-puts-safety-at-the-heart-of-driverexperience/

Isaac, M. (2016, October 28). Twitter's 4-year odyssey with the 6-second video app Vine. The New York Times. https://www.nytimes.com/2016/10/29/technology/twitters-4-year-odysseywith-the-6-second-video-app-vine.html

Keane, M., \& Zhao, E. J. (2012). Renegades on the frontier of innovation: The shanzhai grassroots communities of Shenzhen in China's creative economy. Eurasian Geography and Economics, 53(2), 216-230.

Kelly, M. (2019, December 5). TikTok settles children's data lawsuit one day after it was filed. The Verge. https://www.theverge.com/2019/12/5/20997199/tiktok-bytedance-musicallylawsuit-coppa-settlement-children-data

Kharpal, A. (2020, January 9). A security flaw in China's TikTok app was found. It lets hackers use text messages to control accounts. CNBC Markets. https://www.cnbc.com/ 2020/01/09/tiktok-security-flaw-found-that-allowed-hackers-to-access-accounts.html

Kong, D. (2018). Research report on short video industry. $36 \mathrm{Kr}$ Research Center. http://www.199it.com/archives/672181.html

Leskin, P. (2019, November 6). Employees of TikTok in the US were reportedly pressured to censor "culturally problematic" content that might offend the Chinese government. Business Insider. https://www.businessinsider.com.au/tiktok-china-censorship-employees-restrict-contentdancing-vaping-2019-11?r=US\&IR=T

Li, A. K. (2019). Papi Jiang and microcelebrity in China: A multilevel analysis. International Journal of Communication, 13, 3016-3034.

Light, B., Burgess, J., \& Duguay, S. (2018). The walkthrough method: An approach to the study of apps. New Media \& Society, 20(3), 881-900. https://doi.org/10.1177/1461444816675438

Lin, J., \& de Kloet, J. (2019). Platformization of the unlikely creative class: Kuaishou and Chinese digital cultural production. Social Media + Society, 5(4), 1-12. https://doi.org/ $10.1177 / 2056305119883430$

Lin, T. T. C., Younbo, J., \& Sim, C. (2015). Towards an understanding of intention to use mobile videos: Impression management, perceived facilitation, and social norms. Mobile Media \& Communication, 3(1), 106-124. https://doi.org/10.1177/2050157914555426

Literat, I. (2018). Make, share, review, remix: Unpacking the impact of the internet on contemporary creativity. Convergence, 25(5-6), 1168-1184. https://doi.org/10.1177/ 1354856517751391

Lotz, A. D. (2014). Building theories of creative industry managers. In D. Johnson, D. Kompare, \& A. Santo (Eds.), Making Media Work (pp. 25-38). NYU Press; JSTOR.

McLelland, M., Yu, H., \& Goggin, G. (2017). Alternative histories of social media in Japan and China. In J. Burgess, A. Marwick, \& T. Poell (Eds.), The SAGE handbook of social media (pp. 53-68). SAGE Publications. 
Mehta, S., \& Kaye, D. B. V. (2019). Pushing the next level: Investigating digital content creation in India. Television \& New Media, 1-19. https://doi.org/10.1177/1527476419861698

Meinsenzahl, M. (2019, December 18). TikTok is a "wild west" for influencers. Here are some of the ways they're trying to make money on the viral video app. Business Insider. https://www.businessinsider.com.au/tiktok-stars-are-finding-new-ways-to-monetize-theirvideos-2019-12? $\mathrm{r}=\mathrm{US} \& \mathrm{IR}=\mathrm{T}$

Nagy, P., \& Neff, G. (2015). Imagined affordance: Reconstructing a keyword for communication theory. Social Media + Society, 1(2), 2056305115603385.

Nieborg, D. B. (2015). Crushing Candy: The Free-to-Play Game in Its Connective Commodity Form. Social Media + Society. https://doi.org/10.1177/2056305115621932

Nieborg, D. B., \& Poell, T. (2018). The platformization of cultural production: Theorizing the contingent cultural commodity. New Media \& Society, 20(11), 4275-4292. https://doi.org/ $10.1177 / 1461444818769694$

Parkin, B. (2019, April 17). TikTok: India bans video sharing app. The Guardian. https://www.theguardian.com/world/2019/apr/17/tiktok-india-bans-video-sharing-app

Perez, S. (2019, April 16). Match group restructures exec team with focus on Asia. Tech Crunch. https://techcrunch.com/2019/04/15/match-group-restructures-exec-team-with-focus-on-asia/

Qu, T. (2020, April 30). TikTok and China version Douyin surpass 2 billion download milestone, underlining continued appeal. South China Morning Post. https://www.scmp.com/tech/appssocial/article/3082285/tiktok-and-china-version-douyin-surpass-2-billion-download

Savic, M., \& Albury, K. (2019, July 11). Most adults have never heard of TikTok. That's by design. The Conversation. https://theconversation.com/most-adults-have-never-heard-of-tiktok-thatsby-design- 119815

Sharma, N. (2019, December 11). After ban over child porn, TikTok is going 'woke.' Scroll.In. https://scroll.in/article/946381/after-ban-over-child-porn-tiktok-is-going-woke

Sheng, W. (2020, March 31). ByteDance may now be worth $\$ 100$ Billion. Tech Node. https://technode.com/2020/03/31/bytedance-may-now-be-worth-100-billion/

Sherman, J. (2020, April 2). Unpacking TikTok, mobile apps and national security risks. Lawfare. https://www.lawfareblog.com/unpacking-tiktok-mobile-apps-and-national-security-risks

Silviana, C., \& Potkin, F. (2018, July 11). Indonesia overturns ban on Chinese video app TikTok. Reuters. https://www.reuters.com/article/us-indonesia-bytedance/indonesia-overturns-ban-onchinese-video-app-tik-tok-idUSKBN1K10A0

Spangler, T. (2016, September 30). Musical.ly's Live.ly is now bigger than Twitter's Periscope on iOS (study). Variety. https://variety.com/2016/digital/news/musically-lively-bigger-thanperiscope-1201875105/

Steinberg, M., \& Li, J. (2017). Introduction: Regional platforms. Asiascape: Digital Asia, 4(3), 173-183. https://doi.org/10.1163/22142312-12340076

$\mathrm{Su}, \mathrm{C}$. (2019). Changing dynamics of digital entertainment media in China [Doctoral dissertation, Queensland University of Technology]. https://eprints.qut.edu.au/130744/

Tan, C. K. K., Wang, J., Wangzhu, S., Xu, J., \& Zhu, C. (2020). The abject as mass entertainment: Micro-celebrities in China's Kuaishou video-sharing app. Mobile Media \& Communication, 1 17. https://doi.org/10.1177/2050157920904980

Taylor, J. (2020, July 16). There are calls to ban TikTok in Australia - but you should worry about Facebook too. The Guardian. https://www.theguardian.com/technology/2020/ jul/16/there-are-calls-to-ban-tiktok-in-australia-but-you-should-worry-about-facebook-too

Tidy, J. (2019, December 3). TikTok changes virtual gifts policy after BBC probe. BBC News. https://www.bbc.com/news/technology-50651125

TikTok. (2020). Virtual Items Policy. TikTok. https://www.tiktok.com/legal/virtualitems?lang=en

Tung, H., \& Zhang, Z. (2017, October 31). My conversation with Zhang Yiming, founder of Toutiao. Tech Node. https://technode.com/2017/10/31/hans-tung-toutiao-interview/

Van Dijck, J., \& Poell, T. (2013). Understanding social media logic. Media and Communication, $1(1), 2-14$.

van Dijck, J., Poell, T., \& de Waal, M. (2018). The platform society: Public values in a connective world. Oxford University Press. 
Wang, W. Y., \& Lobato, R. (2019). Chinese video streaming services in the context of global platform studies. Chinese Journal of Communication, 12(3), 356-371. https://doi.org/ 10.1080/17544750.2019.1584119

Wetmore, B., \& Spritz, C. (2019, December 6). The "For You" age: How TikTok conquered 2019. Paper Mag. https://www.papermag.com/tiktok-break-the-internet-2641402771. html?rebelltitem=30\#rebelltitem 30

Xue, Y. (2020, April 27). Tencent-backed Kuaishou launches short video app for global audience - and it looks similar to TikTok. South China Morning Post. https://www.scmp.com/tech/appssocial/article/3081773/tencent-backed-kuaishou-launches-short-video-app-global-audience

Yang, P., \& Tang, L. (2018). "Positive Energy": Hegemonic intervention and online media discourse in China's Xi Jinping era. China: An International Journal, 16(1), 1-22.

Yeung, E. (2019). China Internet Report 2019. abacus, South China Morning Post. https://www.scmp.com/china-internet-report

Zeng, J. (2020). \#MeToo as connective action: A study of the anti-sexual violence and anti-sexual harassment campaign on Chinese social media in 2018. Journalism Practice, 14(2), 171-190. https://doi.org/10.1080/17512786.2019.1706622

Zhang, X., Xiang, Y., \& Hao, L. (2019). Virtual gifting on China's live streaming platforms: Hijacking the online gift economy. Chinese Journal of Communication, 12(3), 340-355. https://doi.org/10.1080/17544750.2019.1583260

Zhang, Z. (2020). Infrastructuralization of TikTok: Transformation, power relationships, and platformization of video entertainment in China. Media, Culture \& Society, 1-18. https://doi.org/10.1177/0163443720939452

\section{Author biographies}

D. Bondy Valdovinos Kaye is an editorial assistant for the Media Industries Journal and a PhD candidate in the Digital Media Research Centre at the Queensland University of Technology. His research interests include digital music, cultural policy, copyright, and platform studies.

Xu Chen is currently an Assistant Professor at the School of Journalism and Communication, Xiamen University, China. His research interests include platform studies, digital cultures, and race, ethnicity and sexuality. Recent publications include peer-review journal articles on Information, Communication \& Society, and Chinese Journal of Communication. hichenxu@hotmail.com

Jing Zeng is a senior research associate in the science communication division in the Department of Communication and Media Research (IKMZ), University of Zurich. Her research interests include digital culture, misinformation, and online activism. 